\title{
Progress Towards Hepatitis A Control and Prevention Through 2019: the National Immunization Program of China
}

\author{
Xiaojin Sun ${ }^{1}$; Fuzhen Wang'; Guomin Zhang'; Hui Zheng'; Ning Miao'; Zundong Yin'; Zhijie An ${ }^{1}{ }^{1, *}$
}

Hepatitis $A$ is an infectious liver disease caused by hepatitis A virus (HAV) that results in mild to severe illness. HAV is spread to immunologically naïve persons who ingest food or water contaminated with feces from HAV-infected individuals (1). Hepatitis A is often seen in sporadic cases, but is also seen in large outbreaks, such as the 1988 outbreak in Shanghai that caused over 300,000 cases and huge economic losses (2). In the pre-vaccine 1970s, China was highly endemic for HAV with a seroprevalence due to infection of $85 \%-95 \%$ in children aged $10-15$ years. With China's socioeconomic development and improvements in standard of living through the 1990s, HAV exposure and hepatitis A incidence shifted from a younger population to an older population (3). Following hepatitis A (HepA) vaccine development, licensure, and widespread introduction, China made great progress in hepatitis A control and prevention. In this report, we describe and interpret a comprehensive analysis of progress towards hepatitis A control and prevention through 2019 from the perspective of China's National Immunization Program.

\section{ACHIEVING HIGH HEPA VACCINE COVERAGE}

Domestically-developed live-attenuated and inactivated HepA vaccines were licensed in China in 1992 and 2002. Annual production of HepA vaccines increased steadily; between 1992 and 2007, 156 million doses of HepA were distributed, mainly for vaccinating schoolaged children (4). Before 2008, all HepA vaccines were non-program vaccines, paid for out-of-pocket by families. Vaccination coverage was relatively low and varied geographically. Coverage was around $16 \%$ to $21 \%$ among those born before 2000 but was growing fast, reaching $53.8 \%$ in western provincial-level administrative divisions (PLADs) and $80.2 \%$ in eastern PLADs among the 2000-2007 birth cohorts.

In 2008, live and inactivated HepA vaccines were both integrated into the Expanded Program on Immunization (EPI). PLADs could select which vaccine to use, and most chose the live-attenuated HepA vaccine with its one-dose schedule at 18 months. A few PLADs selected the inactivated HepA vaccine with its two-dose schedule at 18 and 24 months. Nationally, coverage increased rapidly, reaching $88.1 \%-94.9 \%$ in all 3 regions of China among the 2008-2012 birth cohorts (Figure 1)(5).

Successful immunization programs ensure uniformly high HepA vaccine coverage, regardless of regional socioeconomic development. High coverage following HepA introduction into EPI was seen in other countries such as the United States and Israel (6-7).

\section{POPULATION IMMUNITY FROM VACCINATION}

In a 2006 serological survey, anti-HAV seroprevalence was shown to be increasing with age and was relatively low among children (8). Seroprevalence among children aged 2-14 years was significantly higher in the more developed eastern PLADs than in the developing central and western PLADs, which was consistent with varying vaccine coverage by regions $(8-9)$.

In 2014, after HepA vaccines had been included in the national program in all 3 regions for 6 years, a serological survey showed that anti-HAV seroprevalence among children aged 2-4 years was much higher and had little regional variation (Figure 2). However, seroprevalence was lower among individuals aged 15-19 years than other age groups, indicating an immunity gap among individuals aged 15-19 years. This immunity gap has been described in two other studies $(10-11)$ and is a result of a rapid decrease in hepatitis A incidence following national HepA vaccine introduction causing a decrease in HAV exposure to those born prior to HepA vaccine introduction. Many in this age group, thus, escaped natural infection due to decreased circulation of HAV 


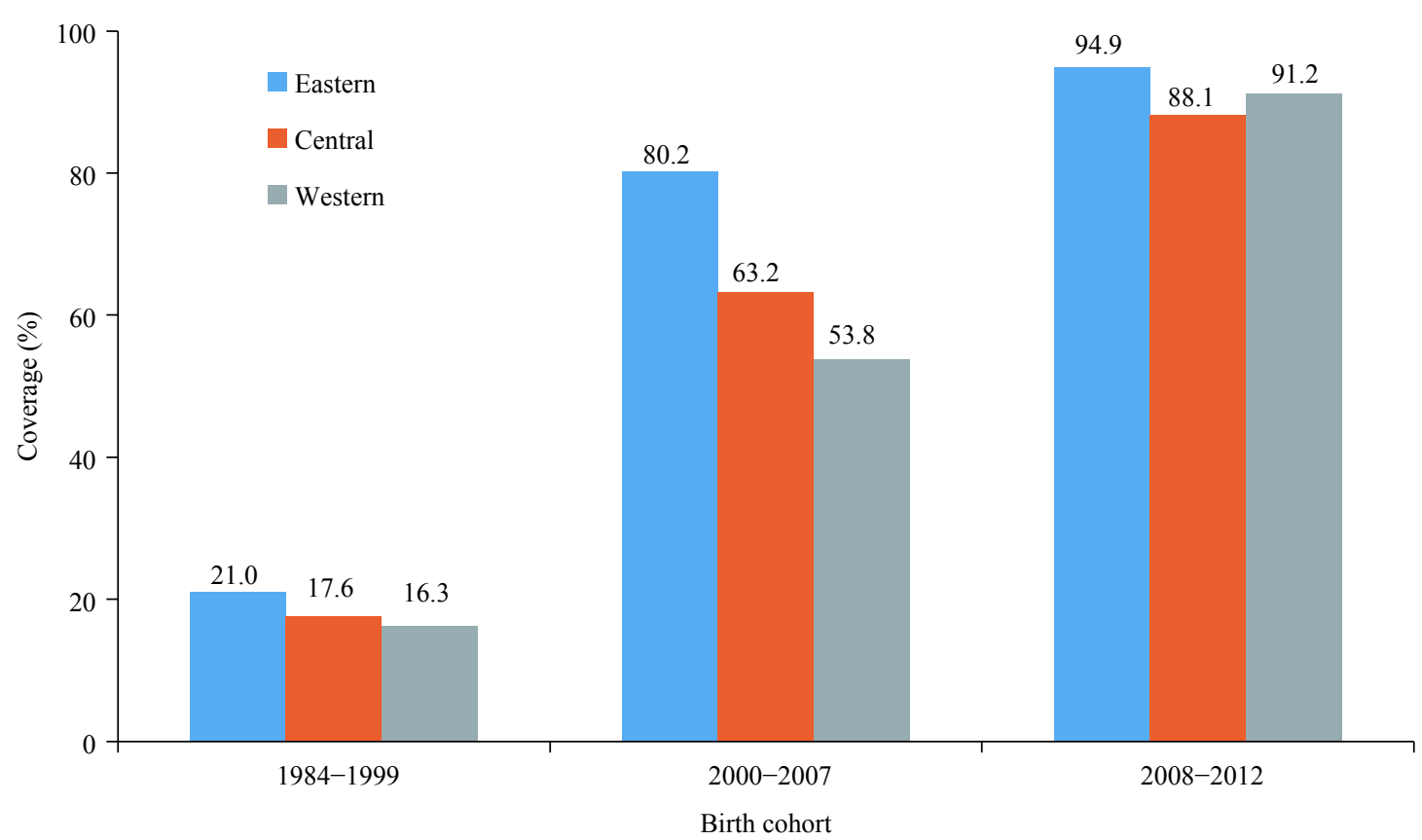

FIGURE 1. Coverage of HepA vaccine by birth cohort and region, China (data from a national serological survey conducted in 2014).

but also had not been immunized by HepA vaccine, which had low coverage before inclusion into EPI.

\section{CONTINUOUS DECREASES IN THE INCIDENCE OF HEPATITIS A}

Hepatitis A incidence is closely related to both sanitation and vaccination. In China and abroad, declines in the incidence of hepatitis A have been well documented among vaccine-targeted populations, but incidence has also been shown to decline among nonvaccine-targeted populations due to indirect protection, e.g. through herd immunity $(6-7,9)$. With better sanitation and widespread use of HepA vaccines in China, HAV-infection-induced immunity has been replaced by vaccine-induced immunity (8). Comparing 2004-2007 (pre-EPI) with 2008-2011, hepatitis A incidence decreased in all age groups and decreased further from 2012-2019. Incidence declines were seen in all PLADs, irrespective of region. In the pre-EPI era, hepatitis A incidence peaked at 5-9 years of age, but the peak was nearly eliminated after nationwide HepA vaccine introduction. Incidence declined in all age groups and in all three phases of EPI, and geographic disparities were greatly narrowed (9). (Figure 3).

However, the hepatitis A incidence was high in the western PLADs, up to $4.28 / 100,000$ among children under 5 years, although there were decreases in incidence. Possible factors for the higher incidence include lower EPI performance, less on-time vaccination, failure to vaccinate, and perhaps weaker sanitation in the western PLADs (12).

\section{DISCUSSION AND CONCLUSION}

Study strengths were that the vaccination status of children aged under 15 years was obtained from official vaccination records; there has been long-term, consistent hepatitis A surveillance; and seroprevalence was determined by nationwide serological surveys with national-level laboratory testing. Limitations were that the vaccination status of individuals aged 15-29 years was based on recall; the passive hepatitis A surveillance system underreports mild cases or infections among individuals who did not seek medical attention; and that laboratory tests cannot distinguish between immunity from disease and immunity from vaccination.

The nationwide introduction of the HepA vaccination has eliminated most age and geographic disparities of hepatitis A disease. HepA vaccine introduction into the EPI system reduced disparities in coverage and seroprevalence in all three regions of China.

An immunity gap among older children and young adults indicated a potential risk of outbreaks among individuals who were born in the years immediately 

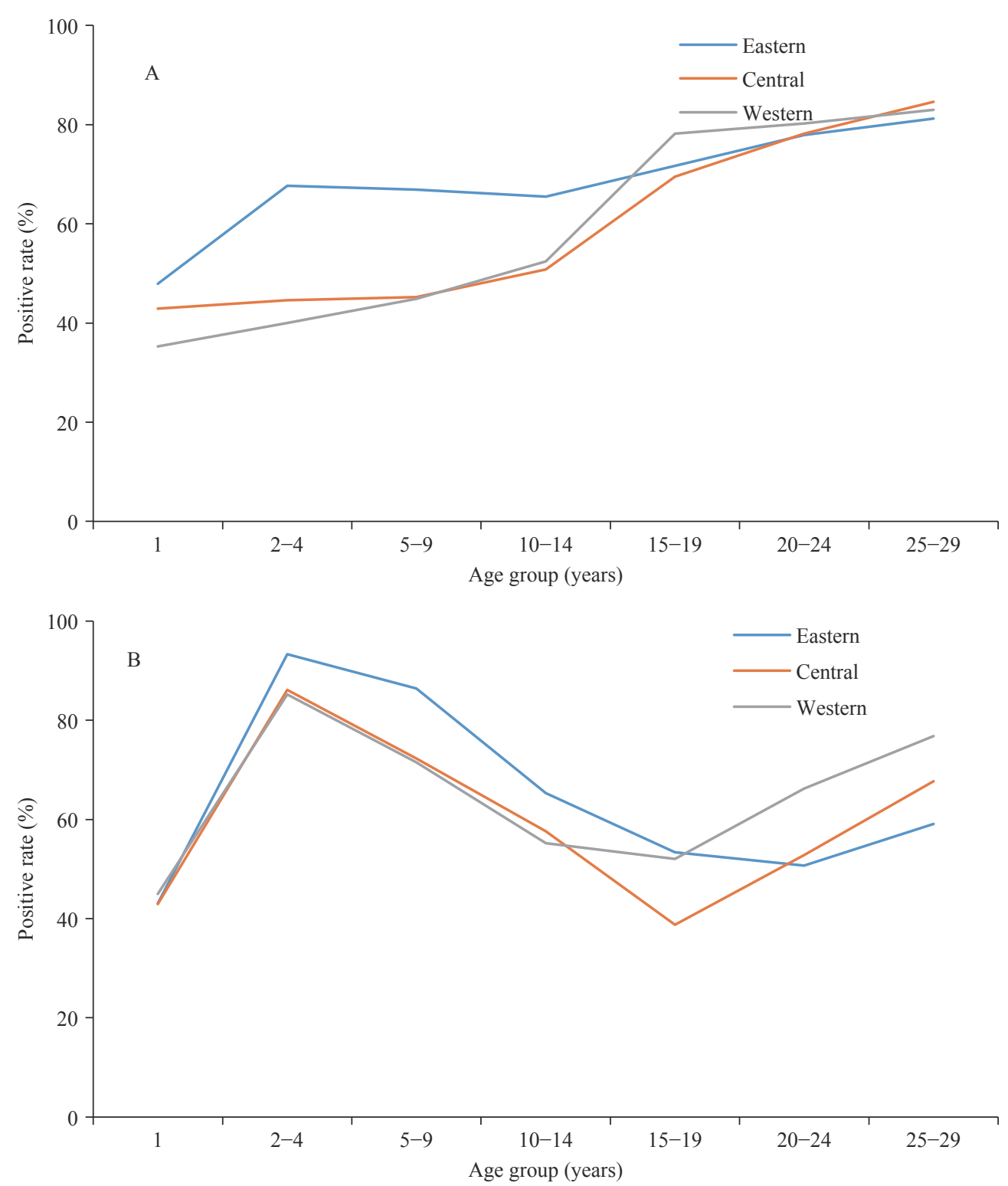

FIGURE 2. Anti-HAV seroprevalence by age and region in 2006 (A) and 2014 (B).

prior to nationwide HepA vaccine introduction. A catch-up vaccination effort could close this immunity gap and should be given serious consideration. An unexpectedly high hepatitis A incidence among children aged 0-4 years in the western PLADs should be closely monitored. Careful evaluation of the western PLADs' immunization programs should be conducted to identify weakness in program performance and means to strengthen the programs.

Maintaining high vaccination coverage among children and strengthening western provincial-level programs will help ensure long-term progress towards elimination of hepatitis A in China.

Acknowledgement: The authors are grateful to the working staff in all levels of CDCs and hospitals that contributed to the prevention and control of hepatitis A in China. Thanks very much to Dr. Lance Rodewald for polishing language.

Conflict of interests: The authors declare no competing interests.

Fundings: The study was funded by the Project of Strengthening National Laboratories, Surveillance \& Program Monitoring Systems, and Service Delivery Models for Viral Hepatitis in China.

doi: $10.46234 / \mathrm{ccdcw} 2020.158$

\#Corresponding author: Zhijie An, anzj@chinacdc.cn.

${ }^{1}$ National Immunization Program, Chinese Center for Disease Control and Prevention, Beijing, China.

Submitted: July 06, 2020; Accepted: July 26, 2020 

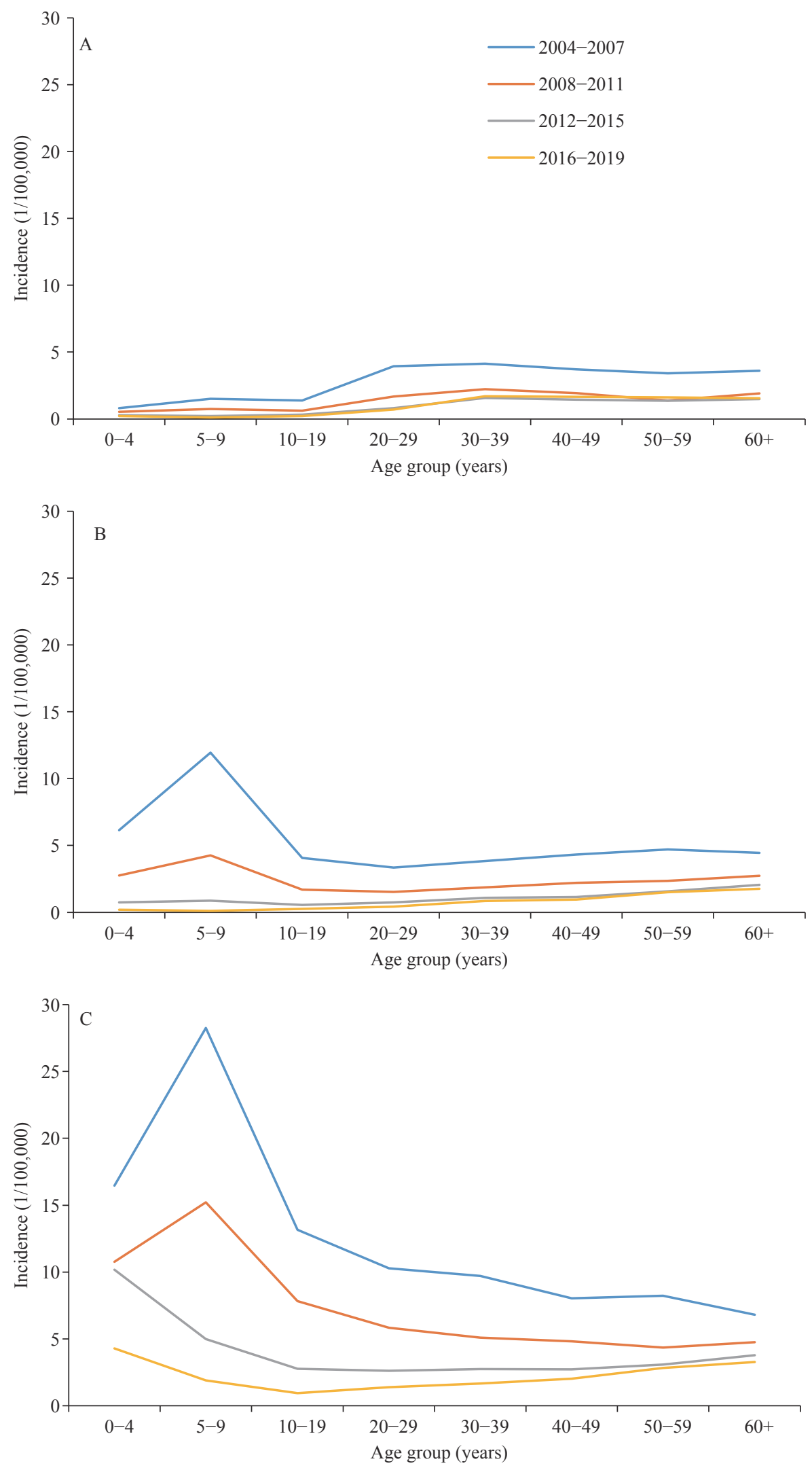

FIGURE 3. Incidence of hepatitis $A$ in three regions in different phases of the expanded program on immunization (EPI). (A) eastern region; $(B)$ central region; $(C)$ western region. 


\section{REFERENCES}

1. WHO position paper on hepatitis A vaccines - June 2012. Wkly Epidemiol Rec 2012;87(28-29):261-76. https://pubmed.ncbi.nlm. nih.gov/22905367/.

2. Tang YW, Wang JX, Xu ZY, Guo YF, Qian WH, Xu JX. A serologically confirmed, case-control study, of a large outbreak of hepatitis A in China, associated with consumption of clams. Epidemiol Infect 1991;107(3):651 - 7. http://dx.doi.org/10.1017/s09502688000 49347.

3. Barzaga NG. Hepatitis A shifting epidemiology in South-East Asia and China. Vaccine 2000;18(S1):S61 - 4. http://dx.doi.org/10.1016/s0264$410 x(99) 00467-3$.

4. Cui FQ, Hadler SC, Zheng H, Wang FZ, Wu ZH, Hu YS, et al. Hepatitis A surveillance and vaccine use in China from 1990 through 2007. J Epidemiol 2009;19(4):189 - 95. http://dx.doi.org/10.2188/ jea.je20080087.

5. Sun XJ, Wang FZ, Zheng H, Miao N, Yuan QL, Cui FQ, et al. The impact of expanded program on immunization with live attenuated and inactivated Hepatitis A vaccines in China, 2004-2016. Vaccine 2018;36(10):1279 - 84. http://dx.doi.org/10.1016/j.vaccine.2018.01. 043.

6. Levine H, Kopel E, Anis E, Givon-Lavi N, Dagan R. The impact of a national routine immunisation programme initiated in 1999 on Hepatitis A incidence in Israel, 1993 to 2012. Euro Surveill 2015;20(7):3 - 10. http://dx.doi.org/10.2807/1560-7917.es2015.20.7. 21040 .
7. Murphy TV, Denniston MM, Hill HA, McDonald M, Klevens MR, Elam-Evans LD, et al. Progress toward eliminating hepatitis a disease in the United States. MMWR Suppl 2016;65(1):29 - 41. http://dx.doi.org/ 10.15585/mmwr.su6501a6.

8. Wang FZ, Sun XJ, Wang F, Zheng H, Jia ZY, Zhang GM, et al. Changing epidemiology of Hepatitis A in China: evidence from three national serological surveys and the National Notifiable Disease Reporting System. Hepatology 2020. http://dx.doi.org/10.1002/hep. 31429.

9. Sun XJ, Zhang GM, Zhou RJ, Zheng H, Miao N, Yin ZD, et al. Changes in the epidemiology of hepatitis $\mathrm{A}$ in three socio-economic regions of China, 1990-2017. Infect Dis Pov 2019;8(1):80. http://dx.doi.org/10.1186/s40249-019-0591-z.

10. Wang H, Gao P, Chen WX, Bai S, Lv M, Ji WY, et al. Changing epidemiological characteristics of Hepatitis A and warning of Anti-HAV immunity in Beijing, China: a comparison of prevalence from 1990 to 2017. Hum Vacc Immun 2019;15(2):420 - 5. http://dx.doi.org/ 10.1080/21645515.2018.1529128

11. Yan BY, Lv JJ, Liu JY, Feng Y, Wu WL, Xu AQ, et al. Changes in seroprevalence of hepatitis A after the implementation of universal childhood vaccination in Shandong Province, China: a comparison between 2006 and 2014. Int J Infect Dis 2019;82:129-34. http://dx.doi.org/10.1016/j.ijid.2019.03.005.

12. Yan HK, Guan J, Wushouer F. Hepatitis a vaccination and antibody levels among children aged 0-6 years in Xinjiang Uygur Autonomous Region, 2013. Chin J Vacc Immun. 2018;24(1):27-31. http://d. wanfangdata.com.cn/periodical/zgjhmy201801007. (In Chinese). 